Article available at nitp://WWw.parasite-journal.org or nitp://dx.dol.org/10.1051/parasite/1995023289

\title{
Phlébotomes (Diptera - Psychodidae) du Sénégal. Peuplements du Ferlo. Isolement d'arbovirus.
}

\author{
TROUILLET J.*, BA Y.**, TRAORE-LAMIZANA M.**, ZELLER H.G. ${ }^{* * *} \&$ FONTENILLE D.**
}

Summary : Phlebotomine SAND Flies (DiPTERA, PSychodidae) From SENEGAl. COMMUNITIES From tHe FERLO AREA. ARBOVIRUSES ISOLATION

Eleven phlebotomine sand flies species were captured in the Ferlo sahelian region during monthly surveys from April 1992 to June 1993. Around the Niakha ground pool, the most well studied location, the most abundant species were respectively Sergentomyia dubia $(40,71 \%)$, S. schewtzi $(25,54 \%)$ and S. clydei $(13,84 \%)$. In total, 98,73\% of the specimens captured belonged to the genus Sergentomyia, and only 1,27\% to the genus Phlebotomus. The sand flies community showed a peak of abundance in January. The sand flies prefered habitats were termite hills, followed by animals burrows and tree holes. In the five communities studied, the most abundant species was $S$. dubia, the gecko leishmaniasis vector. P. duboscai, the human cutaneous leishmaniasis vector, was captured each month, despite its low abundance, except in the ratel burrows. Chandipura and Saboya viruses, as well as two probably new viruses, were isolated from sand flies.

\section{INTRODUCTION}

1 n 1987, une grave épidémie de fièvre de la vallée du Rift s'est déclarée à la frontière sénégalo-mauritanienne (1264 cas dont 224 décès). L'absence d'isolement du virus chez les moustiques, jusqu'à une date récente (Fontenille et al., 1995), et le fait que l'agent de cette arbovirose soit un Phlebovirus (Bunyaviridae), genre dont la plupart des membres sont transmis par des phlébotomes, nous ont amenés à étudier la circulation des arbovirus chez les phlébotomes de la région sahélienne du Ferlo dans le nord du Sénégal, à la limite de l'aire d'épidémicité.

Faute de données sur la faune phlébotomienne de la région, le premier objectif a été de faire l'inventaire et d'étudier les fluctuations saisonnières des populations de ces minuscules diptères.

\footnotetext{
* Département de Biologie animale, Université Cheikh Anta Diop, Dakar, Sénégal.

** Laboratoire de Zoologie médicale, ORSTOM, Dakar, Sénégal.

*** Institut Pasteur, Dakar, Sénégal.

Correspondance : Jean Trouillet, Département de Biologie animale, Université Cheikh Anta Diop, B.P. 5005, Dakar, Sénégal.

Tél. : (221) 257094 - Fax : (221) 324307.
}

\section{Résumé :}

Onze espèces de phlébotomes ont été capturées dans la région du Ferlo. Aux abords de la mare de Niakha, zone la mieux étudiée, les espèces les plus abondantes sont respectivement: Sergentomyia dubia $(40,71 \%)$, S. schwetzi $(25,54 \%)$, S. clydei $(13,84 \%)$. Le genre Sergentomyia constitue $98,73 \%$ des récoltes contre seulement 1,27\% pour le genre Phlebotomus. L'ensemble du peuplement a présenté un maximum d'abondance en janvier. Les habitats préférentiels des phlébotomes sont dans l'ordre: les termitières, les terriers, les trous d'arbres. Dans les cinq peuplements observés, l'espèce dominante est $\mathrm{S}$. dubia, vecteur de la leishmaniose du gecko. Quant à P. duboscai, vecteur de la leishmaniose cutanée humaine, malgré des effectifs faibles, sauf dans les terriers de ratels où il est bien représenté $(18,18 \%)$, il a été récolté chaque mois. Les virus Chandipura et Saboya, ainsi que deux virus probablement nouveaux, ont été isolés.

MOTS CLÉS : phlébotomes, écologie. arbovirus. Afrique de l'Ouest. Sénégal. KEY WORDS : phlebotomine sandflies, ecology. arboviruses. West Africa. Senegal.

Parallèlement, la recherche d'arbovirus a été entreprise.

\section{MATÉRIELS ET MÉTHODES}

\section{RÉGION ÉTUDIÉE}

T e Ferlo est une vaste région sous-peuplée, constituée de plateaux arides, qui couvre près du tiers du territoire sénégalais (fig. 1).

Le climat sahélien, nettement continental, est caractérisé par une grande sécheresse, l'évaporation potentielle dépassant largement la pluviométrie. La hauteur des précipitations, très variable d'une année à l'autre, a été de $420 \mathrm{~mm}$ en 1991, $285 \mathrm{~mm}$ en 1992, $353 \mathrm{~mm}$ en 1993. La courte saison des pluies ou hivernage se situe de juillet à octobre (fig. 2, C). Les températures sont en permanence élevées, janvier étant le mois le plus frais $\left(23^{\circ} \mathrm{C}\right)$ et mai le plus chaud $\left(31^{\circ} \mathrm{C}\right)$; le fléchissement dû aux précipitations (juillet, août, septembre) est suivi d'une brusque remontée quand les pluies s'interromptent (octobre, novembre). L'alizé continental ou harmattan prédomine pendant toute la saison sèche, la mousson apporte les nuages chargés d'humidité de l'hivernage. 


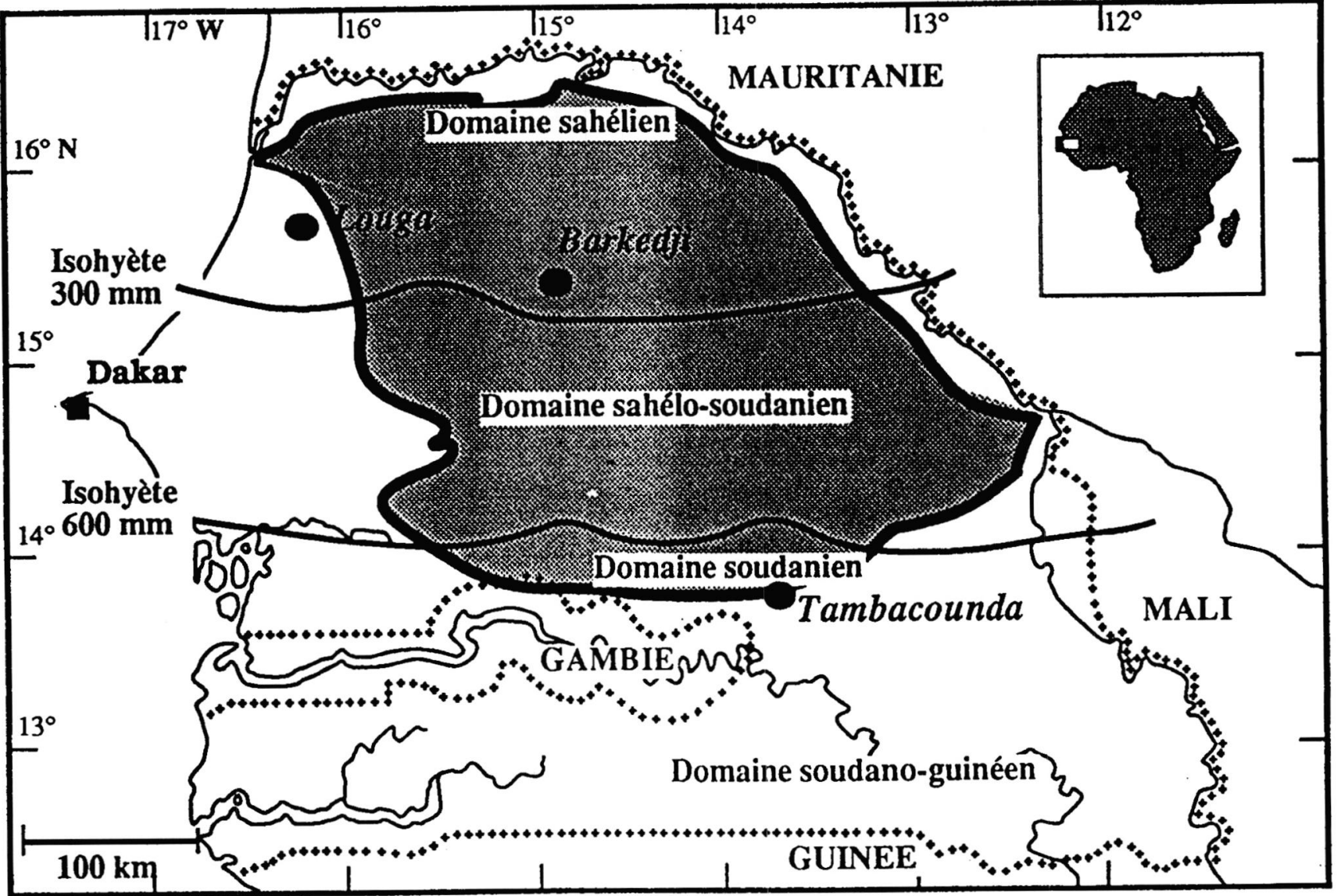

Fig. 1 - La région naturelle du Ferlo

\begin{tabular}{|c|c|c|c|c|c|c|c|c|c|c|}
\hline \multirow{3}{*}{ Espèces } & \multicolumn{6}{|c|}{ Biotopes prospectés } & \multicolumn{4}{|c|}{ Ensemble des biotopes } \\
\hline & \multicolumn{2}{|c|}{ Termitières } & \multicolumn{2}{|c|}{ Terriers } & \multicolumn{2}{|c|}{ Trous d'arbres } & \multirow[b]{2}{*}{ Nb. Phl. } & \multicolumn{3}{|c|}{ Abondance (\%) } \\
\hline & Nb. Phl. & $\begin{array}{c}\text { Abondance } \\
\text { (\%) }\end{array}$ & Nb. Phl. & $\begin{array}{c}\text { Abondance } \\
(\%)\end{array}$ & Nb. Phl. & $\begin{array}{c}\text { Abondance } \\
(\%)\end{array}$ & & Espèces & Sous-Genre & Genre \\
\hline P. duboscqi & 36 & 1,45 & 15 & 1,47 & 7 & 0,65 & 58 & 1,27 & $\begin{array}{c}\text { Phlebotomus } \\
\mathbf{1 , 2 7}\end{array}$ & $\begin{array}{c}\text { Pblebotomus } \\
\mathbf{1 , 2 7}\end{array}$ \\
\hline S. ghesquierei & 0 & 0,00 & 1 & 0,10 & 0 & 0,00 & 1 & 0,02 & Grassomyia & \\
\hline S. inermis & 4 & 0,16 & 1 & 0,10 & 1 & 0,09 & 6 & 0,13 & 1,81 & \\
\hline S. squamipleuris & 58 & 2,34 & 11 & 1,08 & 7 & 0,65 & 76 & 1,66 & & \\
\hline S. adleri & 9 & 0,36 & 0 & 0,00 & 0 & 0,00 & 9 & 0,2 & Sintonius & \\
\hline S. clydei & 439 & 17,70 & 99 & 9,70 & 96 & 8,89 & 634 & 13,84 & 14,04 & Sergentomyia \\
\hline S. antennata & 234 & 9,43 & 82 & 8,04 & 80 & 7,41 & 396 & 8,64 & & 98,73 \\
\hline S. buxtoni & 121 & 4,88 & 27 & 2,65 & 29 & 2,68 & 177 & 3,86 & Sergentomyia & \\
\hline S. dubia & 761 & 30,67 & 499 & 48,92 & 605 & 56,02 & 1865 & 40,71 & 78,75 & \\
\hline S. schwetzi & 744 & 29,99 & 234 & 22,94 & 192 & 17,78 & 1170 & 25,54 & & \\
\hline S. magna & 75 & 3,02 & 51 & 5,00 & 63 & 5,83 & 189 & 4,13 & $\begin{array}{c}\text { Parrotomyia } \\
\mathbf{4 , 1 3}\end{array}$ & \\
\hline Total & 2481 & & 1020 & & 1080 & & 4581 & & & \\
\hline
\end{tabular}

Nb. Phl. $=$ Nombre de phlébotomes

Tableau I. - Effectif et abondance relative des espèces en fonction des biotopes et dans l'ensemble des biotopes 
La végétation est de type savane arbustive sahélienne, caractérisée par des espèces ligneuses, souvent rabougries et épineuses. Des prairies estivales se développent dès les premières pluies.

\section{Biotopes INVENTORIÉS ET TECHNIQUES DE PIÉGEAGE}

Les récoltes de phlébotomes ont été effectuées en grande partie sur le pourtour de mares temporaires proches du village de Barkedji $\left(15^{\circ} 17^{\prime} \mathrm{N}, 14^{\circ} 53^{\prime} \mathrm{W}\right)$ (fig. 1). Ces mares se remplissent avec les pluies et s'assèchent complètement en janvier, février ou mars, selon la réserve d'eau recueillie.

La mare temporaire de Niakha, située à $4 \mathrm{~km}$ à l'ouest du village de Barkedji a été le principal lieu d'étude. Sur son pourtour avaient été déterminées 14 stations de capture constituées soit par des termitières, soit par des terriers de rongeurs ou de reptiles, soit par des trous d'arbres.

Les captures ont été réalisées mensuellement (deux nuits) d'avril 1992 à juin 1993.

Essentiellement, deux types de piégeage ont été utilisés :

- des feuilles de papier blanc enduites d'huile de ricin placées à l'entrée des gîtes de phlébotomes le soir vers $18 \mathrm{~h}$ au coucher du soleil, et relevées le lendemain matin vers $7 \mathrm{~h}$, pour étudier les fluctuations saisonnières et comparer l'attrait des différents biotopes; - des pièges lumineux de type CDC avec carboglace pour obtenir les importantes récoltes nécessaires à la recherche des arbovirus.

Quatre autres zones ont été prospectées de manière plus épisodique :

- la mare de Béliboda, à $7 \mathrm{~km}$ au sud-est de Barkedji;

- la mare de Mogré, à 15 km à l'ouest de Barkedji;

- un champ de terriers de ratels, bordant la piste qui mène à la mare de Béliboda, $5 \mathrm{~km}$ au sud-est de Barkedji. Le ratel (Fissipède, Mustélidé) est un animal robuste et trapu, ayant la taille et l'aspect du blaireau d'Europe, son poids atteint $11 \mathrm{~kg}$;

- les abords immédiats du village de Barkedji.

\section{MONTAGE}

Les phlébotomes recueillis dans ces stations, à l'aide des papiers huilés, ont fait l'objet de montage permanent au baume du Canada, après éclaircissement dans une solution de potasse à $20 \%$, rinçage à l'eau distillée, mordançage dans le liquide de Marc André, déshydratation par passages successifs dans l'alcool à $70^{\circ}, 95^{\circ}$, et la créosote de hêtre.

\section{ISOLEMENT ET IDENTIFICATION DES ARBOVIRUS}

Les lots de phlébotomes constitués à partir des captures aux pièges lumineux sont broyés dans du milieu de Hanks albumine, centrifugés et inoculés à des souriceaux nouveau-nés, des lignées cellulaires Vero et AP 61 d'Aedes pseudoscutellaris. Les virus sont identifiés par immunofluorescence à partir de pools d'ascites immunes de référence et/ou par le test de fixation du complément puis séroneutralisation.

\section{RÉSULTATS}

PEUPLEMENT PHLÉBOTOMIEN DE LA MARE DE

NIAKHA

Richesse et abondance

Il a été capturé dans cette station 4581 phlébotomes appartenant à 11 espèces : Phlebotomus (Phlebotomus) duboscqi Neveu-Lemaire, 1906, Sergentomyia (Grassomyia) ghesquierei (Parrot, 1929), Sergentomyia (Grassomyia) inermis (Theodor, 1938), Sergentomyia (Grassomyia) squamipleuris (Newstead, 1912), Sergentomyia (Sintonius) adleri (Theodor, 1933), Sergentomyia (Sintonius) clydei (Sinton, 1928), Sergentomyia (Sergentomyia) antennata (Newstead, 1912), Sergentomyia (Sergentomyia) buxtoni (Theodor, 1933), Sergentomyia (Sergentomyia) dubia (Parrot, Mornet et Cadenat, 1945), Sergentomyia (Sergentomyia) schwetzi (Adler, Theodor et Parrot, 1929), Sergentomyia (Parrotomyia) magna (Sinton, 1932).

Les espèces du sous-genre Sergentomyia constituent $78,75 \%$ des captures, et le genre Sergentomyia $98,73 \%$ (tableau I). Les espèces les plus abondantes sont respectivement : S. dubia (40,71\%), S. schwetzi $(25,54 \%)$, S. clydei $(13,84 \%)$.

Cet ordre est respecté dans les trois biotopes inventoriés. Toutefois, alors que dans les termitières $S$. schwetzi $(29,99 \%)$ est très proche de $S$. dubia $(30,67 \%)$, la prééminence de cette dernière espèce s'affirme $(48,62 \%)$ dans les terriers et s'accentue $(56,02 \%)$ dans les trous d'arbres. Dans le même temps, les autres principales espèces ( $S$. schwetzi, $S$. clydei, $S$. antennata) sont moins bien représentées dans ces deux biotopes. Il n' y a guère que $S$. magna dont l'abondance varie parallèlement à celle de $S$. dubia.

La densité (nombre de phlébotomes $/ \mathrm{m}^{2}$ de piège) permet de mettre en évidence les gîtes préférentiels de ces insectes (tableau II). Pour l'ensemble du peuplement phlébotomien, ce sont les termitières $(242,28$ phlébotomes $/ \mathrm{m}^{2}$ ) qui se placent en tête devant les terriers $\left(182,14\right.$ phlébotomes $\left./ \mathrm{m}^{2}\right)$ et les trous d'arbres $\left(122,73\right.$ phlébotomes $\left./ \mathrm{m}^{2}\right)$. S. dubia et $S$. magna ont leur densité maximale dans les terriers, $S$. schwetzi, $S$. clydei, S. antennata, S. buxtoni, P. duboscqi, dans les 


\begin{tabular}{|c|c|c|c|c|c|c|}
\hline \multirow[b]{2}{*}{ Espèces } & \multicolumn{2}{|c|}{ Termitières } & \multicolumn{2}{|c|}{ Terriers } & \multicolumn{2}{|c|}{ Trous d'arbres } \\
\hline & Nb. Phl. & $\begin{array}{l}\text { Densité* } \\
\left(\mathrm{phl} / \mathrm{m}^{2}\right)\end{array}$ & Nb. Phl. & $\begin{array}{l}\text { Densité* } \\
\left(\mathrm{phl} / \mathrm{m}^{2}\right)\end{array}$ & Nb. Phl. & $\begin{array}{l}\text { Densité } \\
\left(\mathrm{phl} / \mathrm{m}^{2}\right)\end{array}$ \\
\hline P. duboscqi & 36 & 3,51 & 15 & 2,68 & 7 & 0,79 \\
\hline S. ghesquierei & 0 & 0,00 & 1 & 0,18 & 0 & 0,00 \\
\hline S. inermis & 4 & 0,39 & 1 & 0,18 & 1 & 0,11 \\
\hline S. squamipleuris & 58 & 5,66 & 11 & 1,96 & 7 & 0,79 \\
\hline S. adleri & 9 & 0,88 & 0 & 0,00 & 0 & 0,00 \\
\hline S. clydei & 439 & 42,87 & 99 & 17,68 & 96 & 10,91 \\
\hline S. antennata & 234 & 22,85 & 82 & 14,64 & 80 & 9,09 \\
\hline S. buxtoni & 121 & 11,82 & 27 & 4,82 & 29 & 3,29 \\
\hline S. dubia & 761 & 74,32 & 499 & 89,11 & 605 & 68,75 \\
\hline S. schwetzi & 744 & 72,66 & 234 & 41,78 & 192 & 21,82 \\
\hline S. magna & 75 & 7,32 & 51 & 9,11 & 63 & 7,16 \\
\hline Total & 2481 & 242,28 & 1020 & 182,14 & 1080 & 122,73 \\
\hline
\end{tabular}

* Termitières : 128 pièges ou 10,24 $\mathrm{m}^{2}{ }^{*}$ Terriers : 70 pièges ou 5,60 $\mathrm{m}^{2}-{ }^{*}$ Trous d'arbres : 110 pièges ou $8,80 \mathrm{~m}^{2}-\mathrm{Nb}$. Phl. = Nombre de phlébotomes.

Tableau II. - Densité des différentes espèces et du peuplement dans les trois biotopes prospectés.

termitières. Les trous d'arbres ne recueillent la préférence d'aucune espèce.

Fréquence

Selon le pourcentage de présences de chaque espèce par rapport au nombre de séances de capture effectuées (= nuits) (tableau III), on peut dire que :

- S. dubia, S. clydei, S. schwetzi sont des espèces très communes (fréquence supérieure à $50 \%$ ) dans les trois biotopes;

- $S$. buxtoni est très commune dans les termitières et les terriers, et commune (fréquence comprise entre 25 et $50 \%$ ) dans les trous d'arbres;

- $S$. magna et $S$. antennata sont très communes dans les termitières et communes dans les terriers et les trous d'arbres;

- P. duboscqi et $S$. squamipleuris sont très communes dans les termitières, communes dans les terriers et rares (fréquence comprise entre 10 et $25 \%$ ) dans les trous d'arbres;

- S. inermis est rare dans les termitières et très rare (fréquence inférieure à $10 \%$ ) dans les terriers et les trous d'arbres; $S$. adleri est rare dans les termitières et

\begin{tabular}{|l|r|r|r|}
\hline \multirow{2}{*}{ Espèces } & \multicolumn{3}{|c|}{ Fréquence (\%) } \\
\cline { 2 - 4 } & Termitières & Terriers & Trous d'arbres \\
\hline P. duboscqi & 64,3 & 32,1 & 10,7 \\
S. ghesquierei & 0 & 3,6 & 0 \\
S. inermis & 14,3 & 3,6 & 3,6 \\
S. squamipleuris & 60,7 & 32,1 & 14,3 \\
S. adleri & 21,4 & 0 & 0 \\
S. clydei & 100 & 85,7 & 82,1 \\
S. antennata & 53,6 & 39,3 & 25 \\
S. buxtoni & 96,4 & 53,6 & 46,4 \\
S. dubia & 96,4 & 100 & 92,9 \\
S. schwetzi & 89,3 & 85,7 & 75 \\
S. magna & 60,7 & 46,4 & 42,9 \\
\hline
\end{tabular}

Tableau III. - Féquence dans les trois biotopes prospectés à Niakha. absente ailleurs; enfin, $S$. ghesquierei est très rare dans les termitières et absente ailleurs.

Fluctuations saisonnières

La courbe d'abondance (fig. 2, A) présente un pic aux formes arrondies de novembre à mars, passant par un maximum en janvier, le mois le plus froid. À partir d'avril, les populations de phlébotomes vont aller en diminuant et n'amorceront une légère remontée qu'en août.

S. dubia, S. schwetzi, S. clydei, S. buxtoni, les espèces les plus abondantes, ont été les seules présentes chaque mois :

- S. dubia, l'espèce la plus abondante et la plus fréquente, a présenté son maximum d'activité en janvier-février, et son minimum pendant l'hivernage (juillet, août, septembre), ralentissement qui s'est prolongé en octobre. Sa population s'est ensuite développée à partir de novembre, puis a décliné après le pic signalé (fig. 2, B);

- S. schwetzi a montré un clocher très marqué en décembre, puis a régressé au fur et à mesure que la sécheresse s'est accentuée (fig. 2, B);

- l'activité de $S$. clydei a été plus irrégulière (fig. 2, $\mathrm{B})$;

- la population de S. buxtoni s'est maintenue à un niveau faible; septembre, décembre et janvier étant les mois où cette espèce a été le mieux représentée.

$P$. duboscqi a toujours été pris en petit nombre mais n'a été absent qu'en janvier.

\section{Peuplement PhlÉBotomien de la MARE DE}

BÉLIBODA

Les captures ont eu lieu pendant la même période à l'exception d'avril et de mai 1993, mois pour lesquels il n'y a pas eu de récoltes. 
Nombre de phlébotomes
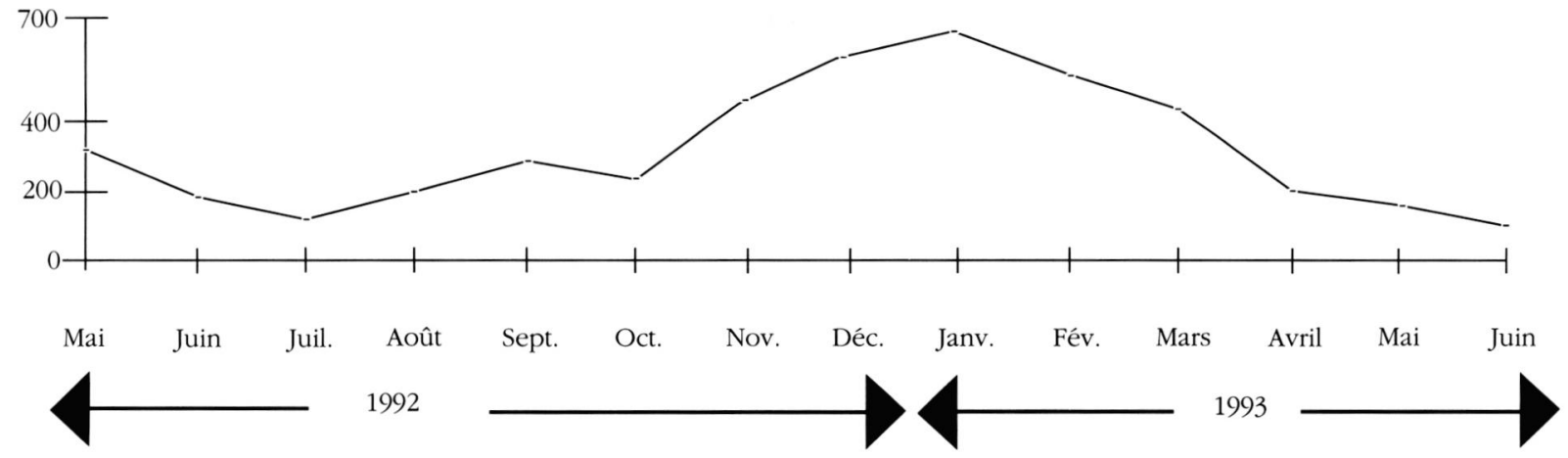

B

Nombre de phlébotomes
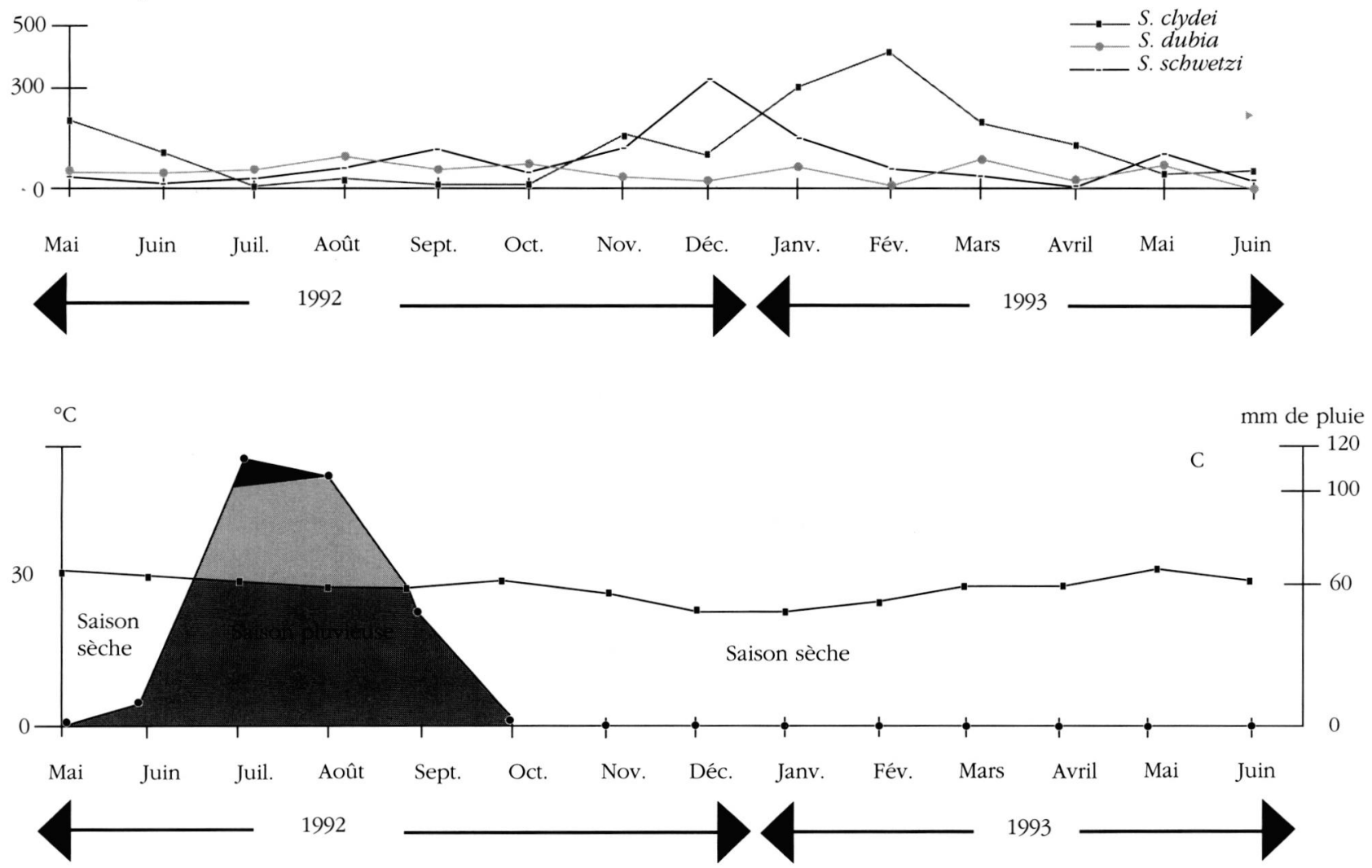

Fig. 2. - A. Fluctuations du peuplement de Niakha; B. Fluctuations saisonnières des principales espèces; C. Diagramme ombrothermique de Barkedji.

Richesse et abondance

Les 2284 phlébotomes capturés appartiennent à neuf espèces : $P$. duboscqi, $S$. inermis, $S$. squamipleuris, $S$. clydei, S. antennata, S. buxtoni, S. dubia, S. schwetzi, S. magna.

Les espèces les plus abondantes sont dans l'ordre : $S$. dubia $(72,59 \%)$, S. antennata (9,68\%), S. clydei (8,76\%), S. schwetzi (5,69\%).
Fréquence

S. clydei (83,33\%), S. dubia (75,00\%), S. schwetzi $(66,67 \%)$ sont très communes. S. antennata, S. buxtoni, $S$. magna sont communes avec la même fréquence de $41,67 \%$.

Les autres espèces sont rares, dont $P$. duboscqi $(16,67 \%)$. 
Fluctuations saisonnières

L'ensemble du peuplement est passé par un maximum très net en décembre.

\section{Peuplement PhLÉBOTOMIEN DE MOGRÉ}

Ce peuplement a été étudié pendant une période plus courte, de janvier à mars 1993.

Richesse et abondance

Parmi les 844 phlébotomes récoltés, six espèces seulement ont été identifiées : S. squamipleuris, S. clydei, S. antennata, S. dubia, S. schwetzi, S. magna.

S. dubia (91,94\%) est l'espèce dominante, elle écrase les autres espèces. Seule l'abondance de $S$. clydei mérite d'être mentionnée $(6,04 \%)$, les autres n'ont été prises qu'en quelques exemplaires.

Fréquence

Les espèces très communes de ce peuplement sont : S. dubia (100\%), S. clydei. (80\%), S. antennata (60 \%), S. schwetzi (60\%).

S. squamipleuris est une espèce commune (40\%) et S. magna une espèce rare $(20 \%)$.

\section{PEUPLEMENT PHLÉBOTOMIEN DES TERRIERS DE} RATELS

Ces terriers ont été prospectés pendant quatre mois, de décembre 1992 à mars 1993.

Richesse et abondance

Les 165 phlébotomes recueillis se rangent en huit espèces : P. duboscqi, S. squamipleuris, S. clydei, S. antennata, S. buxtoni, S. dubia, S. schwetzi, S. magna.

S. dubia est encore la plus abondante $(22,42 \%)$, mais de justesse, suivie de $S$. clydei $(21,21 \%), P$. duboscqi $(18,18 \%)$, S. magna (12,73\%), S. schwetzi (11,51\%).

Fréquence

Pendant cette courte période, sept espèces se sont révélées très communes : $P$. duboscqi $(100 \%), S$. clydei (100\%), S. magna (100\%), S. dubia (75\%), S. buxtoni (75\%), S. schwetzi (50\%), S. antennata (50 $\%)$.

La huitième, S. squamipleuris (25\%) est commune.

\section{Peuplement PhlÉBOtomien du VILLAGE DE \\ BARKEDJI}

Quelques captures ont été effectuées aux abords du village de Barkedji en décembre 1992.

Il a été répertorié six espèces pour 228 phlébotomes récoltés : S. clydei, S. antennata, S. buxtoni, S. dubia, S. schwetzi, S. magna.
Là encore c'est S. dubia qui domine $(77,19 \%)$, devant S. clydei (11,40\%) et $S$. schwetzi (4,38\%). Les trois autres espèces n'ont été représentéees que par quelques individus.

\section{RECHERCHE D'ARBOVIRUS}

Les phlébotomes capturés au piège lumineux de type CDC avec carboglace (33 917 spécimens) ont été testés, sans détermination spécifique préalable, pour recherche d'arbovirus (Fontenille et al., 1994).

À ce jour, trois souches du virus Chandipura, 29 souches du virus Saboya, ainsi que trois souches de deux virus probablement nouveaux ont été isolées.

Le virus Chandipura est un Vesiculovirus (Rhabdoviridae) qui a été isolé de phlébotomes en Inde et d'hommes malades en Inde et au Nigeria (Dhanda et al., 1970). Quant au virus Saboya, c'est un Flavivirus (Flaviviridae) déjà isolé de rongeurs au Sénégal.

\section{DISCUSSION}

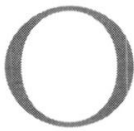

nze espèces de phlébotomes, parmi les 29 répertoriées au Sénégal (Trouillet \& Faye, 1993), ont été capturées au cours de nos prospections dans le Ferlo.

Autour de la mare de Niakha, zone la mieux observée, le genre Sergentomyia constitue 98,73\% des récoltes pour seulement $1,27 \%$ au genre Phlebotomus, uniquement représenté par $P$. duboscqi, le vecteur de la leishmaniose cutanée humaine au Sénégal (Dedet et al., 1978). Ces résultats peuvent être rapprochés de ceux de Basimike et al. (1992), dans la localité de Marigat au Kenya, où les récoltes sont composées de $97,3 \%$ d'individus du genre Sergentomyia pour $2,7 \%$ d'individus du genre Phlebotomus. Il faut par ailleurs remarquer la rareté des espèces du sous-genre Grassomyia (tableau I) qui s'explique par le piégeage adhésif utilisé, leurs gîtes de repos se situant essentiellement dans la végétation herbacée (Trouillet \& Vattier-Bernard, 1990). Des piégeages lumineux ont montré que ce sousgenre était abondant alors que dans le même temps il était peu présent sur les papiers huilés (Ba, 1994).

Dans les cinq peuplements analysés, l'espèce dominante est Sergentomyia dubia qui transmet Sauroleishmania senegalensis (Ranque, 1973), agent de la leishmaniose du gecko (Desjeux \& Waroquy, 1981a).

Des trois biotopes étudiés, ce sont les termitières qui accueillent le plus grand nombre de phlébotomes, devant les terriers et les trous d'arbres. L'utilisation de la densité, nombre de phlébotomes récoltés par unité de surface de piège, permet une comparaison pré- 
cise. Raymond \& Cornet (1976) signalent déjà que, dans la région de Kédougou (Sénégal oriental), les phlébotomes sont les insectes hématophages les plus fréquents dans les termitières-cathédrales. Par contre, Dedet et al. (1978, 1980), Desjeux \& Waroquy (1981b) trouvent les termitières de la région de Thiès (Keur Moussa à $50 \mathrm{~km}$ de Dakar) et de la vallée du fleuve Sénégal assez peu peuplées, alors que les terriers et les trous d'arbres fournissent les récoltes les plus variées et les plus abondantes. Dans le nord du Nigeria, Asimeng (1992) effectue des enquêtes sur le terrain afin de déterminer les habitats naturels prédominants des phlébotomes; les termitières et les creux de rochers sont alors identifiés comme habitats majeurs, les termitières étant les plus importants; les creux d'arbres, les terriers et une grotte constituent des habitats moins fréquentés. Pour Basimike et al. (1992), déjà cités précédemment, les gîtes les plus habités par les phlébotomes sont les termitières, les trous d'arbres et les terriers. Ces trois types de biotopes apparaissent donc comme les gites préférentiels des phlébotomes, l'un ou l'autre étant prépondérant, selon les régions. Il va de soi que ces cavités naturelles offrent à ces petits diptères un environnement très favorable : humidité élevée, température aux fluctuations très atténuées, hôtes variés (crapauds, lézards, serpents, rongeurs, carnivores), autant de conditions qui en font des lieux de prédilection pour le repos et la reproduction de ces insectes.

La profusion des gîtes que nous venons d'évoquer permet aux phlébotomes de trouver, à certains moments, des refuges où ils peuvent se protéger des rigueurs extérieures et rencontrer toutes les conditions propices à l'accomplissement de leur cycle vital. Aussi, la plupart des phlébotomes sont présents toute l'année. La courbe d'abondance du peuplement de la mare de Niakha présente un pic de novembre à mars avec un maximum en janvier, le mois le plus froid, ce qui peut paraître surprenant mais suggère cependant une explication. En effet, en saison des pluies, essentiellement de juillet à début octobre, nombre de gîtes sont lessivés par les précipitations, et ce n'est que vers la fin octobre que les pontes vont pouvoir à nouveau se développer sans trop d'aléas, donnant une première génération d'adultes 30 à 50 jours plus tard (Trouillet \& Vattier-Bernard, 1984), soit fin novembre - début décembre. Ces derniers vont trouver de meilleures conditions climatiques, davantage d'hôtes, et être à l'origine d'une deuxième génération plus nombreuse en janvier. À ce moment-là, les conditions extérieures sont moins favorables, la régression du peuplement débute et s'amplifie vers le mois d'avril quand l'hygrométrie décroissante va limiter certaines populations. Les principales espèces du peuplement suivent approximativement cette fluctua- tion. Toutefois, des variantes peuvent apparaître entre espèces très proches, comme par exemple $S$. dubia et $S$. antennata. Cette dernière est très abondante en fin de saison des pluies (septembre-octobre) avec des effectifs supérieurs à ceux de $S$. dubia, l'espèce dominante, ensuite sa population diminue peu à peu jusqu'à disparaître en fin de saison sèche pour ne réapparaître qu'avec les pluies. S. antennata craint donc davantage la sécheresse que $S$. dubia. $P$. duboscqi, malgré des effectifs faibles, a toujours été récolté.

Pour ce qui est des arbovirus, l'étape suivante consistera à s'intéresser particulièrement aux phlébotomes réputés mammophiles, essentiellement $P$. duboscqi, $S$. clydei, S. schwetzi (Abonnenc, 1972) et à déterminer avec précision la ou les espèces vectrices des virus isolés.

\section{REMERCIEMENTS}

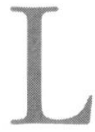

es auteurs remercient le Docteur Digoutte, Directeur de l'Institut Pasteur de Dakar, et Monsieur le Sous-Préfet de Barkedji pour le soutien constant apporté au cours de cette étude, ainsi que Monsieur Malick Faye pour sa collaboration technique lors du montage des phlébotomes.

Ce travail a été financé par l'Institut Français de Recherche Scientifique pour le Développement en Coopération (ORSTOM) et par l'Institut Pasteur de Dakar.

\section{RÉFÉRENCES}

Abonnenc E. Les Phlébotomes de la région éthiopienne (Diptera, Psychodidae). Mémoire ORSTOM, 1972, 55, $289 \mathrm{p}$.

Asimeng E.J. Natural habitats of phlebotomine sandflies in northern Nigeria. Insect Science and its Application, 1992, 13, 113-119.

BA Y. Dynamique des populations de phlébotomes du Ferlo : implication dans la transmission des arbovirus. Mémoire de D.E.A. de Biologie animale, Université Cheikh Anta Diop de Dakar, 1994, 56, 86 p.

Basimike M., Mutinga M.J. \& Kumar R. Habitat preference and seasonal variations of phlebotomine sandflies (Diptera, Psychodidae) in Marigat area, Baringo District, Kenya. Insect Science and its Application, 1992, 13, 307314.

Dedet J.P., Derouin F. \& Cornet M. Infestation spontanée de Phlebotomus duboscqi par des promastigotes de Leishmania au Sénégal. Comptes Rendus des Séances de l'Académie des Sciences de Paris, 1978, sér. D, 286, 301302. 
Dedet J.P., Winshall R., Hayes R.D. \& Desjeux P. Les Phlébotomes (Diptera, Psychodidae) de la vallée du fleuve Sénégal. Première mention de Sergentomyia (Parvidens) lesleyae Lewis \& Kirk, 1946 en Afrique de l'Ouest. Annales de Parasitologie Humaine et Comparée, 1980, 55, 125-133.

Desjeux P. \& Waroquy L. Mise en évidence du cycle évolutif de la leishmaniose du Gecko Tarentola annularis (Geoffroy Saint-Hilaire, 1823) au Sénégal. Rôle vecteur de Sergentomyia dubia (Parrot, Mornet \& Cadenat, 1945). Afrique Médicale, 1981a, 19, 439-442.

Desjeux P. \& Waroquy L. Etude entomologique de 3000 Phlébotomes (Diptera, Psychodidae) du Sénégal. Infestation spontanée par Trypanosomatidae. Afrique Médicale, 1981b, 20, 347-352.

Dhanda V., Rodriges F.M., Ghosh S.N. Isolation of Chandipura virus from sandflies in Aurangabad. Indian Journal of Medical Research, 1970, 58, 179-180.

Fontenille D., Traore-Lamizana M., Trouillet J., Leclerc A., Mondo M., BA Y., Digoutte J.P. \& Zeller H.G. First isolations of arboviruses from phlebotomine sand flies in West Africa. American Journal of Tropical Medicine and Hygiene, 1994, 50, 570-574.

Fontenille D., Traore-Lamizana M., Zeller H.G., Mondo M., Diallo M. \& Digoutte J.P. Rift Valley fever in West Africa : isolations from Aedes mosquitoes during an interepizootic period. American Journal of Tropical Medicine and Hygiene, 1995, 52, 403-404.

Raymond H.L. \& Cornet M. : Phlébotomes (Diptera, Psychodidae) de termitières du Sénégal oriental. Annales de Parasitologie Humaine et Comparée, 1976, 51, 259262.

Trouillet J. \& Faye O. Phlébotomes du Sénégal. Présence de Pblebotomus (Pblebotomus) bergeroti Parrot, 1934 (Diptera, Psychodidae). Annales de Parasitologie Humaine et Comparée, 1993, 68, 101-103.

Trouillet J. \& Vattier-Bernard G. : Elevages de phlébotomes africains (Diptera, Psychodidae). Bulletin de la Société Zoologique de France, 1984, 109, 71-85.

Trouillet J. \& VAtTier-Bernard G. : Les phlébotomes du Congo (Diptera, Psychodidae). Journal of African Zoology, 1990, 104, 477-540.

Accepté le 4 avril 1995 